\title{
Seltene Krankheiten und Orphan-Medikamente: eine Herausforderung für das Gesundheitswesen
}

Romain Lazor ${ }^{a}$,

Loredana D'Amato Sizonenko ${ }^{b}$

a Leitender Arzt, Sprechstunde für interstitielle Pneumopathien und seltene Lungenerkrankungen, Abteilung für Pneumologie, Centre Hospitalier Universitaire Vaudois, Lausanne

b Leitende Ärztin, Koordinatorin Orphanet Schweiz Abteilung für Genetische Medizin, Hôpitaux Universitaires de Genève

Die Auffassung, dass verschiedene seltene Krankheiten gemeinsam als ein Phänomen betrachtet werden können, entstand vor rund 30 Jahren in den USA. Sie ist vor allem auf ein Gesetz zurückzuführen, das auf die Förderung der Entwicklung von Medikamenten für diese Krankheiten ausgerichtet war. Eine seltene Krankheit wurde durch eine Prävalenzschwelle definiert, unterhalb der die Behandlung als unrentabel gelten kann, was somit die Entwicklung und Markteinführung einer Therapie verhindert. Ausgehend von dieser ökonomischen Definition, die auf das Arzneimittel ausgerichtet ist, und vor allem unter dem Einfluss von Patientenorganisationen veränderte sich die Wahrnehmung: Unterdessen stehen bei den seltenen Erkrankungen aus gesellschaftlicher und ethischer Sicht die betroffenen Menschen im Mittelpunkt. Auch wenn der Begriff «seltene Erkrankungen» nicht einem klar umschriebenen wissenschaftlichen und epidemiologischen Konzept entspricht, wurden die mit diesen Krankheiten verbundenen Schwierigkeiten in verschiedenen Ländern als gesundheitspolitisches Problem anerkannt und Massnahmen für dessen Lösung getroffen.

\section{Eine breit angelegte europäische Umfrage hat erstmals die Schwierigkeiten und Bedürfnisse der betroffenen Patienten genau erfasst und gezeigt, dass diese Menschen nicht optimal versorgt werden.}

Korrespondenz:

Dr. med. Romain Lazor Service de pneumologie Policlinique Médicale Universitaire Rue du Bugnon 44 CH-1011 Lausanne romain.lazor@chuv.ch

\section{Ausmass des Problems}

Gemäss der Definition der Europäischen Union gilt eine Erkrankung als selten, wenn sie eine Prävalenz $<1 / 2000$ aufweist. Meist handelt es sich um eine chronische, fortschreitende, invalidisierende Krankheit, die mit einer verkürzten Lebenserwartung verbunden sein kann und aufgrund ihrer tiefen Prävalenz eine komplexe, hochspezialisierte Behandlung erfordert. Von den über 7000 bekannten seltenen Erkrankungen sollen $80 \%$ genetisch bedingt sein. Die Zahl der seltenen Krankheiten nimmt zu, da Erkrankungen, die ursprünglich als ein Krankheitsbild galten, mit dem Bekanntwerden ihrer genetischen Ursachen in eine Vielzahl von Krankheiten unterteilt werden. Insgesamt leiden in Europa schätzungsweise 30 Millionen Menschen an seltenen Erkrankungen, d.h. 5 bis $6 \%$ der Bevölkerung.

\section{Zusammenfassung}

Von seltenen Erkrankungen, die definitionsgemäss eine Prävalenz von unter 1/2000 aufweisen, sind schätzungsweise 5 bis $6 \%$ der Bevölkerung betroffen, d.h. in der Schweiz rund 500000 Menschen. Eine breit angelegte Umfrage hat erstmals die Schwierigkeiten und Bedürfnisse der betroffenen Patientinnen und Patienten genau erfasst und aufgezeigt, dass diese Menschen nicht optimal versorgt werden. In den meisten europäischen Ländern bestehen bereits nationale Pläne für seltene Erkrankungen oder werden in den nächsten Jahren entwickelt. Die Schweiz weist in diesem Bereich einen Rückstand von mehreren Jahren auf, der jedoch aufgeholt werden könnte, falls die kürzlich eingeleiteten Initiativen breite Unterstützung erhalten. Bei Orphan-Medikamenten, die für eine kleine Zahl von Patientinnen und Patienten bestimmt, aber oft sehr teuer sind, kann das Einzelinteresse dem Allgemeininteresse entgegenstehen. Die seltenen Erkrankungen und ihre Behandlung werfen ethische, gesellschaftliche, ökonomische, aber auch wissenschaftliche und klinische Fragen auf und zeigen die Grenzen des Wissens und der Mittel auf. Sie sind ein Problem für das Gesundheitswesen, und eine Herausforderung, der sich die Schweiz stellen sollte.

\section{Schwierigkeiten und Erwartungen der Patientinnen und Patienten}

Alle Menschen mit einer seltenen Erkrankung sehen sich mit ähnlichen Schwierigkeiten konfrontiert: falsche oder verspätete Diagnosestellung, Mangel an Informationen, psychische Belastung durch die Isolation und die fehlende Hoffnung auf Heilung sowie keine praktische Unterstützung im Alltag. Eine Umfrage zu 18 seltenen Erkrankungen, die bei 12000 Betroffenen in 17 europäischen Ländern durchgeführt wurde, hat das Ausmass der Schwierigkeiten und die Bedürfnisse der Patientinnen und Patienten aufgezeigt; sie enthält erstmals auch Daten zur Schweiz [1]. 
Im Durchschnitt benötigten die Patienten neun verschiedene medizinische Leistungen und $43 \%$ mussten im Verlauf der letzten zwei Jahre stationär behandelt werden. $16 \%$ der Patienten hatten mehr als fünf Ärzte konsultiert, bevor die Diagnose gestellt wurde. In $25 \%$ der Fälle lag zwischen dem Auftreten der ersten Symptome und der endgültigen Diagnose ein Zeitraum von 5 bis 30 Jahren. Bei $43 \%$ wurde anfänglich eine falsche Diagnose gestellt. Dies hatte bei 79\% dieser Patienten eine unangemessene Behandlung, darunter bei $16 \%$ chirurgische Eingriffe, zur Folge. In $69 \%$ der Fälle wurde davon ausgegangen, dass die verspätete Diagnose mindestens eine negative Auswirkung hatte (körperliche oder psychische Folgeerscheinungen, unangemessenes Verhalten der Eltern gegenüber ihrem Kind, Geburt eines weiteren kranken Kindes). In $27 \%$ der Fälle wurden die Umstände, unter denen die Diagnose mitgeteilt wurde, als schlecht oder unannehmbar erachtet. $75 \%$ erachteten eine psychologische Unterstützung bei der Aufklärung über die Diagnose als notwendig, aber nur 23\% hatten eine derartige Unterstützung erhalten [1]. Die medizinischen Leistungen entsprachen in $94 \%$ der Fälle den Erwartungen der Patienten, doch der $\mathrm{Zu}$ gang zu diesen Leistungen wurde in $24 \%$ der Fälle als schwierig oder unmöglich beurteilt. Von den 27\% der Patienten, die die Sozialdienste in Anspruch nahmen, waren $48 \%$ sehr oder teilweise zufrieden, 52\% hingegen waren eher oder sehr unzufrieden [1]. Insgesamt entsprechen die schweizerischen Daten in etwa denen der anderen europäischen Länder und
Patientinnen und Patienten an. Im Rahmen des vierten Internationalen Tags der seltenen Krankheiten zum Thema «Ungleiche Gesundheitsversorgung» organisierte ProRaris am 19. Februar 2011 erstmals in der Schweiz einen Informationstag. Diese Veranstaltung, die von über 450 Personen besucht wurde, war ein grosser Erfolg.

\section{Staatliche Massnahmen in Europa und in der Schweiz}

Die Europäische Union und mehrere Mitgliedstaaten haben bereits spezifische Massnahmen zur Bekämpfung der seltenen Krankheiten getroffen, die als bedeutende Herausforderung für das Gesundheitswesen anerkannt werden. $\mathrm{Zu}$ erwähnen sind unter anderem die Verordnung über Arzneimittel für seltene Leiden (1999), drei Programme im Bereich der öffentlichen Gesundheit (1999-2013), spezifische Forschungsbudgets (6. und 7. Forschungsrahmenprogramm der Europäischen Union) sowie 2009 der Erlass der Empfehlung «Seltene Krankheiten: eine Herausforderung für Europa», mit der die einzelnen Mitgliedstaaten aufgefordert werden, bis Ende 2013 einen Plan oder eine nationale Strategie für die seltenen Erkrankungen einzuführen (Europlan) [3]. In diesem Zusammenhang wurde 2010 ein Sachverständigenausschuss (EUCERD) eingesetzt [4]. Erstmals ist die Schweiz darin vertreten.

1997 wurde in Frankreich Orphanet aufgebaut: Dieses Informationsportal für seltene Krankheiten und Orphan Drugs ist unterdessen in fast 40 europä-

\section{Zwar wurden zu den seltenen Krankheiten Einzelinitiativen lanciert, doch hat sich bisher keine schweizerische Institution des Problems als Ganzem angenommen.}

lassen darauf schliessen, dass die Versorgung bei den seltenen Krankheiten nicht optimal ist.

\section{Die Patientinnen und Patienten - «Experten» für ihre Krankheit}

Eine wichtige Rolle zur Verbesserung der Situation von Menschen mit einer seltenen Erkrankung spielen die Patientenorganisationen. Sie können psychologische und soziale Unterstützung bieten, die Isolation durchbrechen, über die Krankheit informieren, Erfahrungen austauschen, praktische Hilfe bieten, die Teilnahme an klinischen Studien fördern oder gar die Forschung finanzieren. Durch den Zusammenschluss zu nationalen Allianzen und/oder europäischen Vereinigungen können diese Organisationen eine politische Wirkung entfalten. Die europäische Allianz Eurordis vereint fast 480 Patientenorganisationen aus 45 Ländern [2]. Der Allianz Seltener Krankheiten Schweiz, der 2010 gegründeten ProRaris, gehören neben 37 Patientenorganisationen auch einzelne ischen Ländern präsent. Orphanet gilt als wichtiges Instrument für die nationalen Strategien zu den seltenen Erkrankungen. Seit April 2011 ist es eine gemeinsame Aktion der Mitgliedstaaten der Europäischen Union, an der sich auch die Schweiz aktiv beteiligt.

Bei der koordinierten Patientenversorgung hat Frankreich eine Pionierrolle übernommen: Der erste nationale Plan für seltene Erkrankungen (2005-2008) mit einem Budget von 108 Millionen Euro [5] sah zehn strategische Stossrichtungen vor, unter anderem den Zugang zur Gesundheitsversorgung, die Versorgungsqualität, die Ausbildung der Fachpersonen, die Förderung der Forschung und den Aufbau von nationalen und europäischen Partnerschaften. Es wurden 131 nationale Referenzzentren und 501 regionale Kompetenzzentren bezeichnet [6]. Soeben wurde der zweite nationale Plan für seltene Erkrankungen (2011-2014) mit einem Budget von 180 Millionen Euro vorgelegt [7].

Ausgehend von den europäischen Daten wurde die Zahl der Menschen mit einer seltenen Erkrankung 
in der Schweiz auf 500000 geschätzt [8]. Zwar wurden zu den seltenen Krankheiten einige Einzelinitiativen lanciert, doch hat sich bisher keine schweizerische Institution des Problems als Ganzem angenommen. Die Schweiz hat 2002 die europäische Definition der seltenen Krankheiten übernommen und 2006 wurde eine Regelung zu wichtigen Arzneimitteln für seltene Krankheiten eingeführt, mit der das Zulassungsverfahren vereinfacht wird. Seit 2001 ist Orphanet Schweiz [9] an den Genfer Universitätsspitälern institutionalisiert, die einen Teil der Aktivitäten finanzieren. Dank der Unterstützung der Kantone und der Schweizerischen Gesundheitsdirektorenkonferenz seit 2007 konnte das Informationsportal Legitimität erwerben. Dennoch erhält das Problem der seltenen Krankheiten weiterhin nur geringe Beachtung. Die Schweiz befindet sich diesbezüglich mehrere Jahre im Rückstand. Referenzzentren bestehen bisher ebenso wenig wie eine spezifische Finanzierung der Forschung durch die Behörden. Im Februar 2010 trafen sich Akteure aus Politik, Verwaltung, Verbänden und Wirtschaft erstmals zu einem Gedankenaustausch zum Thema seltene Erkrankungen und Orphan-Medikamente [10]. Nationalrätin Ruth Humbel reichte im Dezember 2010 ein Postulat ein, das darauf ausgerichtet ist, die gesundheitliche Situation von Menschen mit seltenen Krankheiten zu verbessern [11]. Sie fordert darin, dass in Zusammenarbeit mit den betroffenen Organisationen und Fachpersonen sowie mit den Kantonen eine nationale Strategie für diese Krankheiten erarbeitet wird, damit Betroffene in der ganzen Schweiz medizinisch gleich gut versorgt werden.

\section{Problem der Orphan-Medikamente}

Die Entwicklung von neuen Behandlungen für die seltenen Erkrankungen ist eine entscheidende Herausforderung. 1983 wurde in den USA ein Gesetz zur Förderung der Entwicklung von Orphan-Medikamenten erlassen. Zuvor hatten diese aufgrund der geringen Zahl von Betroffenen als unrentabel gegolten. In der Folge wurden in der Europäischen Union und in weiteren Ländern ähnliche Regelungen erlassen. Um die Pharmaindustrie zur Entwicklung derartiger Medikamente zu veranlassen, sehen diese Gesetze verschiedene finanzielle Anreize sowie ein Alleinvertriebsrecht während sieben bis zehn Jahren vor, was einen Return-onInvestment ermöglicht. Mit diesen Regelungen konnten eindrückliche Fortschritte erzielt werden: Innerhalb von 25 Jahren wurden in den Vereinigten Staaten 370 Orphan-Arzneimittel auf den Markt gebracht; in der Europäischen Union waren es innerhalb von zehn Jahren rund 60 Medikamente. Die Pharmaindustrie ihrerseits hat erkannt, dass die Orphan-Medikamente einen erheblichen Markt darstellen, der zurzeit auf USD 85 Milliarden pro Jahr mit einem jährlichen Wachstum von $6 \%$ geschätzt wird [12].

Es wurden jedoch auch Auswüchse festgestellt, vor allem in England. Einige Pharmaunternehmen haben längst bekannte Heilmittel, für die bereits akademische Institutionen wissenschaftliche Studien durchgeführt hatten, als Orphan-Medikamente angemeldet. Diese Produkte waren nicht vermarktet worden, weil sie finanziell nicht interessant waren, wurden aber von öffentlichen Institutionen zu geringen Kosten lizenzfrei hergestellt. Durch ihre Anmeldung als Orphan-Medikamente konnte ihr Preis massiv erhöht werden, während die Investitionen und Risiken geringer waren als bei einem neuartigen Heilmittel. So kann mit Hydroxyharnstoff, der für die Behandlung der chronischen myeloischen Leukämie zugelassen ist, ein Patient mit Sichelzellanämie für 160 GBP pro Jahr behandelt werden, während der gleiche Wirkstoff 14900 GBP pro Jahr kostet, wenn er als Orphan-Medikament für Sichelzellanämie zugelassen ist [13]. Der Preis für ein Gramm Ibuprofen steigt von 0,8 GBP als Schmerzmittel auf 6575 GBP als Mittel zur intravenösen Behandlung des persistierenden Ductus arteriosus [13]. Die jährlichen Kosten von 3,4-Diaminopyridin zur Behandlung des Lambert-Eaton-Syndroms erhöhten sich entsprechend von 1000 GBP auf 44200 [14] und jene von Carbamylglutamat von 4000 GBP auf 95000 [15]. Angesichts derartiger Preiserhöhungen verweigerten die Versicherer die Kostenübernahme mit dem Argument, das Kosten-NutzenVerhältnis sei unangemessen [14]. Damit war den Patientinnen und Patienten der Zugang zu diesen Arzneimitteln verwehrt, obwohl mit den Gesetzen zu den Orphan-Medikamenten genau das Gegenteil angestrebt wurde. Im November 2010 wurde deshalb der britische Premierminister in einem offenen Brief aufgefordert, Massnahmen zu treffen, um dem massiven Preisanstieg bei den Orphan-Medikamenten zu begegnen [14]. Zudem wurde dazu aufgerufen, die Regelung zu überarbeiten [16]. In Brasilien, wo der Zugang zu Orphan-Medikamenten nicht gesetzlich geregelt ist, wurde in einer Studie untersucht, wie eine Laronidase-Behandlung bei Mukopolysaccharidose vom Typ I (jährliche Kosten 350000 USD) finanziert wurde: $87 \%$ der Patientinnen und Patienten erreichten die Übernahme ihrer Behandlung über Gerichtsprozesse, die gegen den Staat angestrengt wurden [17]. In einer norwegischen Umfrage wurde untersucht, ob die Öffentlichkeit in einem Kontext mit beschränkten Mitteln der Behandlung der seltenen Erkrankungen gegenüber den häufigen Krankheiten den Vorzug gibt [18]. Bei einem Szenario mit gleich hohen Kosten bevorzugten $11 \%$ die seltenen Krankheiten und $25 \%$ die häufigen Krankheiten, die übrigen machten keinen Unterschied. Bei einem Szenario, in dem die Behandlungskosten der seltenen Erkrankungen mit dem Faktor 4 multipliziert wurden, bevorzugten nur 7\% die seltenen Krankheiten, während $45 \%$ den häufigen Erkrankungen den Vorzug gaben [18]. Diese Umfrage legt nahe, dass zwar Bestrebungen bestehen, den seltenen Krankheiten gleich und gerecht zu begegnen, dass ihnen jedoch kaum der Vorzug gegeben wird, wenn ihre Behandlung zulasten der häufigen Krankheiten geht.

In der Schweiz musste sich das Bundesgericht mit einem Rechtsstreit zwischen einer Patientin mit Mor- 
bus Pompe, der mit Myozyme behandelt wurde, und ihrem Krankenversicherer befassen. In diesem $\mathrm{Zu}$ sammenhang vertrat es die Auffassung, von der Grundversicherung könne nicht verlangt werden, dass sie eine Behandlung übernehme, die jährlich $600000 \mathrm{CHF}$ koste (Urteil 9C_334/2010 vom 23. November 2010). Damit führte das Bundesgericht den Begriff der Rationierung ein und bezifferte die maximalen Kosten, die von der sozialen Krankenversicherung für ein zusätzliches Lebensjahr verlangt werden können, auf 100000 CHF. Dieses Urteil, das für einen Einzelfall erlassen wurde, veranlasste Krankenversicherer, ihre Kostengutsprachen für Myozyme-Behandlungen bei anderen Patienten zurückzuziehen [19]. Einer dieser Patienten berichtete am ersten Internationalen Tag der seltenen Krankheiten, der in der Schweiz am 19. Februar 2011 stattfand, über seine Erfahrungen. Dieses Urteil löste verschiedene parlamentarische Vorstösse aus [20, 21, 22] und bewog den Bundesrat, auf dem Verordnungsweg daran zu erinnern, dass die Übernahme eines nicht auf der Liste stehenden Medikaments weiterhin im Einzelfall geprüft werden müsse. Dabei müssten die allgemeine Wirkung des Arzneimittels, seine Wirkung im Einzelfall sowie die Kriterien der Zweckmässigkeit und der Wirtschaftlichkeit berücksichtigt werden. Zusätzlich müsse den Grundsätzen der Verhältnismässigkeit und der Rechtsgleichheit Rechnung getragen werden.

Mit der Entwicklung von neuen Therapien für die seltenen Erkrankungen und der zunehmenden Zahl von Patientinnen und Patienten, die behandelt werden können, wird somit der Preis der Orphan-Medikamente zu einer Frage, bei der sich das Einzelinteresse und das Interesse der Gesellschaft entgegenstehen. In diese Diskussion werden ethische, juristische, ökonomische, gesellschaftliche und politische Argumente eingebracht. Sind Orphan-Medikamente zu billig und unrentabel, werden sie nicht entwickelt. Sind sie zu teuer, besteht die Gefahr, dass sie nicht übernommen werden. In beiden Fällen stehen sie den betroffenen Patientinnen und Patienten nicht zur Verfügung.

\section{Fazit}

Die seltenen Erkrankungen und ihre Behandlung werfen ethische, gesellschaftliche, ökonomische, aber auch wissenschaftliche und klinische Fragen auf und zeigen die Grenzen des Wissens und der Mittel auf. Sie sind ein Problem für das Gesundheitswesen, und eine Herausforderung, der sich die Schweiz stellen sollte. Dabei kann sie auf den Erfahrungen der Nachbarländer aufbauen.

\section{Literatur}

1 Eurordis. The Voice of 12000 Patients. Experiences and expectations of rare disease patients on diagnosis and care in Europe. 2010. www.eurordis.org/IMG/ pdf/voice_12000_patients/EURORDISCARE_ FULLBOOKr.pdf (28.6.2011).

2 www.eurordis.org/ (28.6.2011).
3 http://ec.europa.eu/health/ph_threats/non_com/ docs/rare_com_de.pdf (28.6.2011).

4 www.eucerd.eu/EUCERD/_PP_1.html (28.6.2011).

5 www.orpha.net/actor/Orphanews/2006/doc/ plan_national.pdf (28.6.2011).

6 Referenzzentren mit Qualitätslabel und Kompetenzzentren für die Behandlung von seltenen Erkrankungen. www.orpha.net/orphacom/cahiers/docs/FR/ Liste_des_centres_de_reference_labellises.pdf (28.6.2011)

7 www.orpha.net/actor/Orphanews/2011/doc/ Plan_national_maladies_rares.pdf (28.6.2011).

8 Lazor R, D'Amato Sizonenko L. Seltene Erkrankungen: 30 Millionen Menschen in Europa sind betroffen. Schweiz Ärztezeitung.2008;89(15):636-8.

9 www.orphanet.ch/ (28.6.2011).

10 Oggier W. Seltene Erkrankungen und Orphan Drugs in der Schweiz - Handlungsbedarf aus gesundheitsökonomischer Sicht. Schweiz Ärztezeitung. 2010;91(18):734-5

11 Humbel R. Nationale Strategie zur Verbesserung der gesundheitlichen Situation von Menschen mit seltenen Krankheiten. Postulat 10.4055 vom 16.12.2010; abrufbar unter: www.parlament.ch/d/ suche/seiten/geschaefte.aspx?gesch_id=20104055 (28.6.2011)

12 Sharma A, Jacob A, Tandon M, Kumar D. Orphan drug: Development trends and strategies. J Pharm Bioallied Sci. 2010;2:290-9.

13 Ferner RE, Hughes DA. The problem of orphan drugs. BMJ. 2010;341:c6456.

14 Nicholl DJ, Hilton-Jones D, Palace J, Richmond S, Finlayson S, Winer J, et al. Open letter to prime minister David Cameron and health secretary Andrew Lansley. BMJ. 2010;341:c6466.

15 Leonard JV, Richmond S. Pricing of orphan drugs. Lancet. 2009;373:462.

16 McCabe C, Stafinski T, Menon D. Is it time to revisit orphan drug policies? BMJ. 2010;341:c4777.

17 Boy R, Schwartz IV, Krug BC, Santana-da-Silva LC, Steiner CE, Acosta AX, et al. Ethical issues related to the access to orphan drugs in Brazil: the case of mucopolysaccharidosis type I. J Med Ethics. 2011;37:233-9.

18 Desser AS, Gyrd-Hansen D, Olsen JA, Grepperud S, Kristiansen IS. Societal views on orphan drugs: cross sectional survey of Norwegians aged 40 to 67. BMJ. 2010;341:c4715.

19 Hundsberger T, Rohrbach M, Rösler KM. Die Problematik der Enzymersatztherapie beim Morbus Pompe (Glykogenose Typ II). Eine medizinische Einschätzung. Schweiz Med Forum. 2011;11:427-9.

20 Humbel R. Bundesgerichtsurteil als Startschuss zur medizinischen Rationierung? Interpellation 11.3154 vom 16.3.2011; abrufbar unter: www.parlament. ch/d/suche/seiten/geschaefte.aspx?gesch $\mathrm{id}=20113154$. (28.6.2011).

21 Gutzwiller F. Ein Schwellenwert von 100000 Franken für medizinische Therapien? Interpellation 11.3306 vom 18.3.2011; abrufbar unter: www.parlament. $\mathrm{ch} / \mathrm{d} /$ suche/seiten/geschaefte.aspx?gesch $\mathrm{id}=20113306$. (28.6.2011).

22 Cassis I. Wie viel soll die Gesellschaft für ein Lebensjahr zahlen? Postulat 11.3218 vom 17.3.2011. www.parlament.ch/d/suche/seiten/geschaefte. aspx?gesch_id=20113218. (28.6.2011). 